\title{
Spontaneous Internal Drainage of Iatrogenic Oesophageal Perforation
}

\author{
Ramya Kalaiselvan ${ }^{1}$, Michael Pallot ${ }^{1}$, Vinutha Shetty ${ }^{1}$, Neal Townsend ${ }^{2}$ and Siba Senapati ${ }^{1 *}$ \\ ${ }^{1}$ Department of General Surgery, Salford Royal NHS Foundation Trust, England \\ ${ }^{2}$ Department of Radiology, Salford Royal NHS Foundation Trust, England
}

Received: September 28, 2017; Published: October 11, 2017

*Corresponding author: S Senapati, Consultant Upper Gastrointestinal \& Bariatric Surgeon, Department of General Surgery, Salford Royal NHS Foundation Trust, Manchester M6 8HD, England

\begin{abstract}
Oesophageal perforation is a potentially fatal disease; however, with newer technologies many strategies are available to manage this. We present a case of a 60 year old woman with iatrogenic perforation of Zenker's diverticulum and how she was managed.
\end{abstract}

\section{Introduction}

Oesophageal perforation remains a potentially fatal disease with mortality rates of $10 \%$ to $40 \%$ reported [1]. Iatrogenic injury is the most frequent cause of oesophageal perforation, with studies reporting incidence rates of $14 \%$ - 100\% [2], followed by spontaneous rupture (Boerhaave syndrome) [3]. A variety of treatment options are available ranging from conservative medical therapy to radical oesophagectomy. Newer modalities with interventional endoscopy with or without placement of stents are more frequently being described [4].

\section{Case Report}

60 -year old female with symptoms of dysphagia and reflux underwent diagnostic oesophago-gastroduodenoscopy (OGD). Immediately post-procedure, patient complained of chest pain and shortness of breath. Surgical emphysema was presentin the neckand Computed Tomography (CT) confirmed oesophageal perforation in an upper oesophageal diverticulum (Figure 1). She remained well whilst managemened conservatively for 10 days with nasogastric tube, intravenous antibiotics and omeprazole. Subsequently the patient became septic, developing signs of mediastinitis and lung consolidation. She was transferred to Intensive care unit (ICU) where parenteral nutrition was commenced and antibiotics were changed appropriately. A repeat CT did not demonstrate any collections which required drainage, however there was a large iatrogenic oesophageal diverticulum extending from the retropharyngeal space down to the posterior mediastinum, just beyond the carina, compressing on the oesophagus. The patient also developed a neck swelling and air leak from a suspected perforated pharyngeal pouch for which ENT opinion was sought.

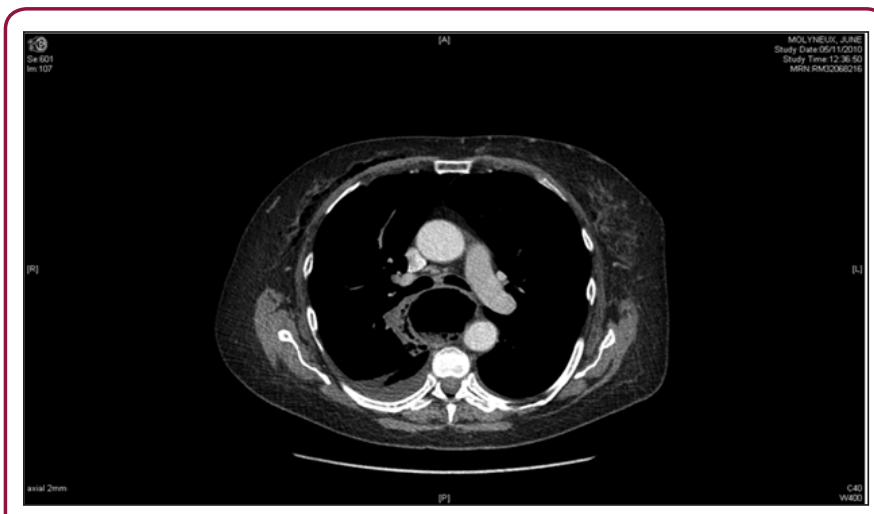

Figure 1.

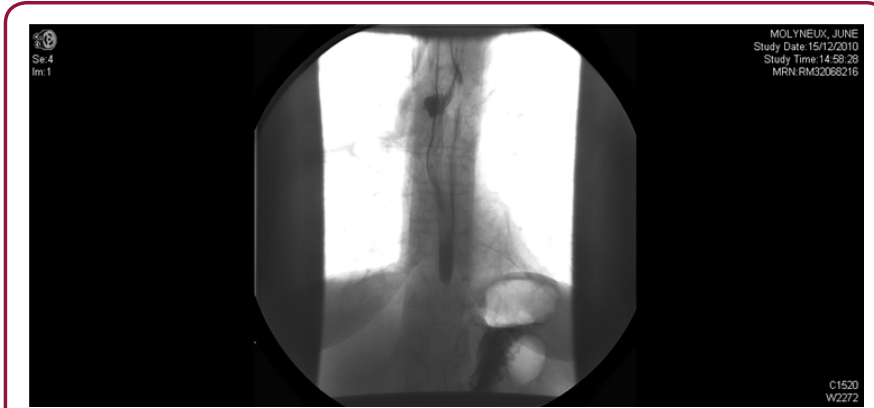

Figure 2.

Repeat CT showed a small amount of contrast, identified within the gas containing mediastinal cavity which suggested a persistent small leak (Figure 2). The patient continued to be septic and did not 
improve as expected. Hence a repeat CT and contrast swallow was performed which demonstrated no leak in the cervical oesophagus, however there was a persistent defect. Contrast was noted to be leaking from a defect at the level of carina (Figure 3).

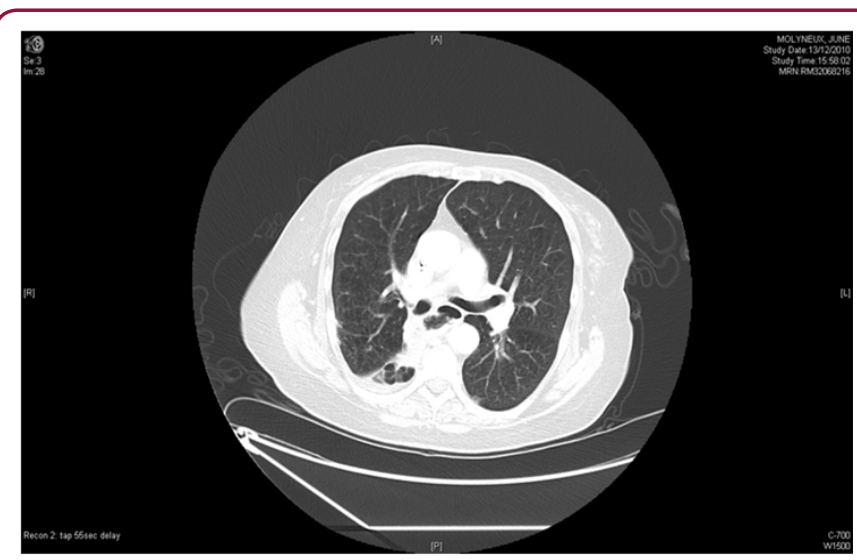

Figure 3.

A feeding jejunostomy was placed and a rigid OGD demonstrated a large defect in the thoracic oesophagus as well as perforated upper oesophageal diverticulum. ENT surgeons excised the diverticulum and drained the cavity. The distal perforation was left alone at this stage. This fortunately resolved spontaneously in 4 weeks without the need for further intervention. The upper oesophageal perforation/abscess cavity had internally drained itself back into the posterior thoracic oesophagus. Treating the proximal perforation led to spontaneous resolution of the distal perforation and abscess cavity.

\section{Discussion}

Flexible video endoscopy has almost totally replaced rigid oesophagoscopy. Despite the inherent safety of the procedure ( $0.03 \%$ perforation risk compared to $0.11 \%$ for rigid endoscopy), the dramatic increase in the number of examinations performed has led to an increase in the number of associated injuries [5]. Perforation related to diagnostic upper GI endoscopy occurs distally in 75 - 90\%, mostly in relation to pathology. Most iatrogenic trauma is recognised immediately, or there is at least a high index of suspicion. Our patient presented soon after the procedure and appropriate management was commenced. However, this failed subsequently and she had to have multiple imaging only to diagnose a second oesophageal defect that was associated with an abscess cavity.
Iatrogenic cervical perforations are almost always contained and thus collections are usually drained percutaneously when necessary. Any resulting oesophagocutaneous fistulas heal rapidly in the absence of distal obstruction. Occasionally operative prevertebral lavage, primary closure and drainage is required, using a left lateral incision anterior to the sternocleidomastoid. This is well tolerated by even critically ill patients [5]. The optimal treatment of oesophageal perforation remains an issue of continuing debate. Surgical intervention has to be weighed against a conservative approach. Traditionally, the surgical approach was reserved to perforations diagnosed within a timeframe of $24 \mathrm{hr}$. If the diagnosis is not made early, surgery carries a high morbidity and mortality. Conservative therapy may be the better choice if the process is limited to the mediastinum, while surgery should be confined to those cases with simultaneous rupture or perforation of the pleura.

Our patient went onto develop a distal oesophageal defect thus internally draining the upper oesophageal perforation. Managing the upper oesophageal perforation lead to resolution and healing of the lower oesophageal defect. This is the first case to be reported in literature to clearly demonstrate that internal drainage and dealing with the proximal perforation could be attempted before contemplating thoracotomy if such a case is encountered in future.

\section{Acknowledgement}

This work was supported by The Departments of Radiology and General Surgery at Salford Royal NHS Trust.

\section{References}

1. Bresadola V, Terrosu G, Favero A, F Cattin, V Cherchi, et al. (2008) Treatment of perforation in the healthy esophagus: analysis of 12 cases. Langen- becks Arch Surg 393(2): 135-140.

2. Vallböhmer D, Hölscher AH, Hölscher M, Bludau M, Gutschow C, et al. (2010) Options in the management of esophageal perforation: analysis over a 12-year period. Dis Esophagus 23(3): 185-190.

3. Chirica M, Champault A, Dray X, Sulpice L, Munoz-Bongrand N, et al. (2010) Esophageal perforations. J Visc Surg 147(3): e117-e128.

4. LA Brewer, R Carter, GA Mulder, Stiles QR (1986) Options in the management of perforations of the esophagus. Am J Surg 152(1): 62-69.

5. Silvis SE, Nebel O, Rogers G, Sugawa C, Mandelstam P (1976) Endoscopic complications. Results of the 1974 American Society for Gastrointestinal Endoscopy Survey 235(9): 928-930.

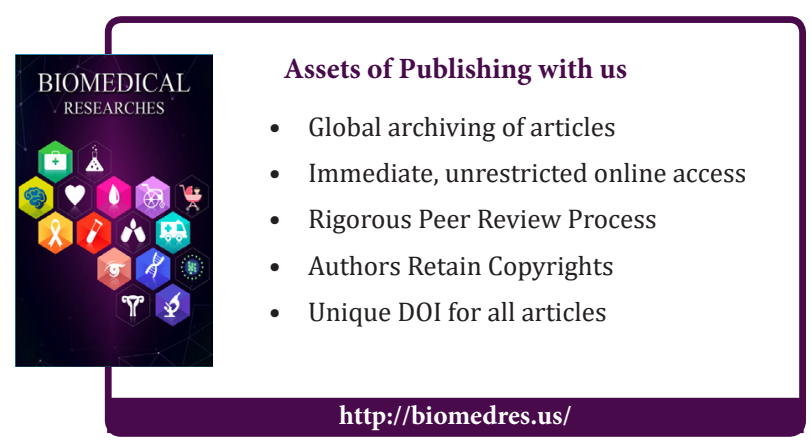

